\title{
Seguridad eléctrica en el lugar de trabajo
}

Frank Neyra Vela ${ }^{1}$

\begin{abstract}
RESUMEN
La electricidad es el motor del progreso en toda industria y la forma de energía más utilizada en el mundo actual; sin embargo, si no es protegida y no se tienen los conocimientos necesarios para manejarla, existe el potencial de que cause daño. La presente investigación abarca los principales riesgos en el manejo de la electricidad: el arco eléctrico y el choque eléctrico. Es por ello que se aplica una metodología en seguridad eléctrica que consiste en reconocer los peligros asociados al uso de la energía eléctrica para tomar acciones preventivas con la finalidad de evitar los riesgos que ocasionan lesiones o muertes. Al realizar un análisis del arco y choque eléctricos, los resultados aportan para la prevención de accidentes dentro del lugar de trabajo, según los estándares de las normas NFPA 70E y IEEE 1584-2002.
\end{abstract}

Palabras claves: seguridad eléctrica; arco eléctrico; choque eléctrico.

\section{INTRODUCCIÓN}

Hoy en día para interactuar con cualquier sistema eléctrico se requiere un conocimiento previo de las normas que establecen los requisitos para el diagnóstico y evaluación de una instalación. Esto tiene por objetivo identificar las condiciones peligrosas, el deterioro físico, la mala utilización y otras situaciones que aumentan la probabilidad de riesgo para las personas y su patrimonio (Carlos y Campos, 2013); es por esta razón que se cuenta con requerimientos de seguridad, a nivel nacional, definidos en el Decreto Supremo n. ${ }^{\circ}$ 024-2016-EM. Reglamento de Seguridad y Salud Ocupacional en Minería (Ministerio de Energía y Minas, 28 de julio de 2016) y en la Resolución Ministerial n. ${ }^{\circ} 111-2013-M E M-D M$. Reglamento de Seguridad y Salud en el Trabajo con Electricidad (Merino, 17 de marzo de 2013).

Es importante conocer la normativa vigente sobre la seguridad eléctrica, especialmente los riesgos de choque y arco eléctricos, los cuales han existido desde que el hombre empezó a utilizar la energía eléctrica. No obstante, el número de muertes, lesiones graves, quemaduras y pérdida de bienes ha aumentado debido a una falta de concientización sobre seguridad eléctrica (Industrial Technology Suppliers, Inc., 2014). La concientización sobre la problemática es una deficiencia que aún se presenta y que puede ser enfocada en los dos estados iniciales de las fases del aprendizaje: la incompetencia-inconsciente y la incompetencia-consciente (Sobrino, 2013); en otras palabras, en los trabajadores que no son conscientes del daño que podrían sufrir y en los que todavía no saben controlarlo. Al no estar informado sobre la problemática, cualquier trabajador de la industria, en general, se expone a peligros eléctricos con un gran potencial de causar daños. Sin embargo, estos peligros se pueden identificar como condiciones subestándares. Actualmente, las mejoras en seguridad requieren cumplir con las condiciones ligadas a los costos, a la burocracia en empresas, al compromiso, al interés de la alta dirección, entre otros; lamentablemente, muchas veces se espera la lesión o muerte de un trabajador para recién adoptar por la

1 Ingeniero industrial. Actualmente, es ingeniero de seguridad y salud ocupacional en la Compañía Minera Antapaccay. (Arequipa, Perú)

ORCID: https://orcid.org/0000-0002-1133-6875

E-mail: Frank.Neyra@glencore.com.pe 
condición más segura en las instalaciones para el empleado.

Sobrino (2013) menciona que la mejora continua y su aplicación pueden solucionar los problemas previstos y la administración de riesgos potenciales, tomando en cuenta que es un ciclo que se debe estar repitiendo constantemente. Asimismo, Niebel y Freivalds (2009) señalan que los accidentes son el resultado de una secuencia de eventos causados por múltiples razones. Por otro lado, con respecto a la teoría de la causalidad de Bird, es importante indicar que esta tiene como propósito neutralizar los efectos destructivos de pérdidas potenciales o reales, las cuales son consecuencia de acontecimientos no deseados; por ello, la correcta aplicación de los conocimientos y técnicas de un profesional en los métodos y procedimientos de un trabajo puede reducir las pérdidas causadas por este tipo de acontecimientos (Vásquez, 27 de marzo de 2017).

Considerando lo anterior, el principal objetivo de este artículo es relacionar las condiciones de trabajo con los riesgos eléctricos; además, se busca ampliar el conocimiento en cuanto a la exposición de riesgos con energía eléctrica para prevenir accidentes. A raíz de estos propósitos, se plantean como objetivos específicos identificar la manera en la que un factor contribuye a la intensidad de energía de un arco y choque eléctrico, y proponer un modo para prevenir o reducir accidentes relacionados a estos riesgos eléctricos. Cabe resaltar que la finalidad de este artículo está alineada con la teoría de Bird, ya que la necesidad de neutralizar las causas y efectos que generan accidentes en la industria debe realizarse mediante la administración de los peligros; es por ello que esta investigación impulsa a aportar soluciones preventivas para evitar accidentes relacionados con la energía eléctrica, incluyendo el uso de los equipos de protección personal y la tecnología que disminuya la severidad del riesgo.

Finalmente, este estudio toma en consideración las contribuciones de trabajos relacionados a la seguridad eléctrica, como el de López (2003), que aporta con el diseño de un programa en Microsoft Visual Basic 6.0 cuyo objetivo es analizar los riesgos eléctricos que suceden dentro de las oficinas y centros de cómputo, por medio de la identificación de peligros y la propuesta de acciones correctivas. Del mismo modo, se toma en cuenta el estudio de Clavijo, Vera y Landín (2009), el cual ayuda a cómo prevenir incendios al analizar los riesgos y peligros en estaciones de transformación de energía y centros de control de motores. A su vez, se consideró la tesis de Rivadeneira y Torres (2010), quienes realizaron un estudio de fallas y control de protecciones de riesgos eléctricos y proponen un enfoque sobre diversos elementos de protección para asegurar la calidad de una instalación eléctrica, mostrando sus principales características, así como los equipos de protección personal obligatorios en el trabajo para conservar la vida de las personas. Asimismo, se revisó el análisis y gestión de riesgos realizado por Serrano (2011) para el mantenimiento de un sistema eléctrico con la metodología HAZOP, el cual busca asegurar posibles fallos que podrían conllevar a problemas económicos, políticos, sociales y ambientales. Finalmente, se examinó el texto de Trejo (2013), en donde se comparan las normas NFPA 70E e IEEE 1584-2002, y se determina la exposición de energía incidente a los trabajadores, encontrando algunos criterios de un sistema eléctrico de potencia industrial en el que la magnitud de la tensión de operación y de la corriente de corto circuito (arco eléctrico) pueden ocasionar daños a las personas; además, se establece la categoría de riesgo según el nivel de energía incidente y se identifican los equipos de seguridad (equipo de protección personal) para evitar accidentes.

\section{METODOLOGÍA}

La investigación es cuantitativa, puesto que se realizaron mediciones numéricas y reportes de lo sucedido. Las principales técnicas utilizadas fueron la entrevista, la observación y el análisis de datos o investigación documental; a continuación se detallan:

- La entrevista se realizó con personal de mantenimiento, con el cual se mantuvo una interacción directa

- La observación fue directa estructurada, porque se utilizó una guía de observación (estructurada), lista de frecuencias, lista de chequeo, registro anecdótico y un diario de campo

- El análisis documental consideró toda fuente de data secundaria, como investigaciones anteriores, libros, informes, reportes, guías, manuales, documentos electrónicos y páginas web, requisitos para la implementación de un sistema de gestión SSO (SGSSO) y normas legales nacionales e internacionales.

Asimismo, se tiene un nivel de investigación explicativa, debido a que se estableció una relación causal; es decir, no solo se buscó describir o acercarse a un problema, sino que se intentó encontrar las causas del mismo. 
Por otro lado, la unidad de análisis correspondió a un grupo de trabajadores del área de mantenimiento eléctrico. En dicha área se desarrolló la medición, la cual fue de interés para el presente artículo.

Con respecto a la metodología del análisis de riesgos, se realizó un estudio de posibilidades y consecuencias de cada factor de riesgos eléctricos (arco y choque eléctrico), de tal forma que se puedan optimizar las condiciones de trabajo y así administrar el riesgo en el nivel más bajo como sea razonablemente factible. Para el riesgo de arco eléctrico, la National Fire Protection Association (2012) en conjunto con el Institute of Electrical and Electronics Engineers (2002) cuentan con métodos de cálculo para la energía incidente y las fronteras de protección; mientras que, para el choque eléctrico, se ha considerado a la ley de Ohm para determinar el paso de la corriente a través del cuerpo en diferentes escenarios.

\section{RESULTADOS}

En cuanto al análisis e interpretación de resultados, se planteó un criterio teórico de contraste para la hipótesis general, la hipótesis específica 1 y la hipótesis específica 2, considerando que el valor $p=$ 0.05 . Luego, cuando se obtenga el valor $p>0.05$, se aceptará como respuesta válida Ho. Pero, si se obtiene el valor $p<0.05$, se aceptará como respuesta válida $\mathrm{Ha}$. El modelo de regresión lineal y correlación de Pearson fue la estadística utilizada para el contraste de la hipótesis.

\section{Planteo de hipótesis general}

Se planteó la siguiente relación con respecto a la hipótesis general:

- Ho: la relación de un análisis de riesgos eléctricos y las condiciones de trabajo no influye en reducir los accidentes eléctricos.

- Ha: la relación de un análisis de riesgos eléctricos y las condiciones de trabajo influye en reducir los accidentes eléctricos.

Tabla 1. Estadística de contraste de la hipótesis general.

\begin{tabular}{|c|c|c|}
\hline Model & $\mathbf{R}$ & Sig. F Change \\
\hline 1 & 0.885 & 0.005 \\
\hline
\end{tabular}

Fuente: Elaboración propia.

Aplicando el software SPSS21, se identificó una correlación conjunta de $R=0.885$ y un valor $p=0.005$ (ver Tabla 1). Entonces, según el criterio teórico, se aceptó que existe una relación significativa, donde las condiciones de trabajo y el análisis de riesgos eléctricos influyen en la reducción de los accidentes eléctricos.

\section{Planteo de hipótesis especifica 1}

Se planteó la siguiente relación con respecto a la hipótesis específica 1 :

- Ho: la relación del valor nominal de un arco eléctrico no influye en la reducción de accidentes eléctricos.

- Ha: la relación del valor nominal de un arco eléctrico influye en la reducción de accidentes eléctricos.

Tabla 2. Estadística de contraste de la hipótesis específica 1.

\begin{tabular}{|l|l|l|}
\hline \multicolumn{3}{|c|}{ Análisis_arco_eléctrico } \\
\hline Pearson correlation & Reducción_Accidente_Eléctrico & 0.646 \\
\hline Sig. (1-tailed) & Reducción_Accidente_Eléctrico & 0.022 \\
\hline N. ${ }^{\circ}$ & Reducción_Accidente_Eléctrico & 10 \\
\hline
\end{tabular}

Fuente: Elaboración propia.

Se identificó una correlación conjunta de $\mathrm{R}=0.646$ y un valor $p=0.022$ (ver Tabla 2). Entonces, según el criterio teórico, se aceptó que existe una relación del valor nominal de un arco eléctrico y que además influye en la reducción de accidentes eléctricos.

\section{Planteo de hipótesis especifica 2}

Se planteó la siguiente relación con respecto a la hipótesis específica 2:

- Ho: la relación de la intensidad de un choque eléctrico no influye en la reducción de accidentes.

- Ha: la relación de la intensidad de un choque eléctrico influye en la reducción de accidentes eléctricos.

Tabla 3. Estadística de contraste de la hipótesis específica 2.

\begin{tabular}{|l|l|l|}
\hline \multicolumn{3}{|c|}{ Análisis_choque_eléctrico } \\
\hline Pearson correlation & Reducción_Accidente_Eléctrico & 0.878 \\
\hline Sig. (1-tailed) & Reducción_Accidente_Eléctrico & 0.000 \\
\hline N. ${ }^{\circ}$ & Reducción_Accidente_Eléctrico & 10 \\
\hline
\end{tabular}

Fuente: Elaboración propia.

Se identificó una correlación conjunta de $\mathrm{R}=0.878$ y un valor $p=0.000$ (ver Tabla 3). Entonces, según el criterio teórico, se aceptó que la relación de la intensidad de un choque eléctrico influye en la reducción de accidentes eléctricos. 


\section{DISCUSIÓN}

El contraste de hipótesis realizado se evidencia en la Tabla 4 y en la Figura 1.

Tabla 4. Valoración de intervalos de correlación de Pearson.

\begin{tabular}{|c|c|}
\hline $\begin{array}{c}\text { Intervalo de correlación de } \\
\text { Pearson }\end{array}$ & Valoración \\
\hline De 0.000 a 0.20 & Baja influencia \\
\hline De 0.201 a 0.40 & Moderado bajo \\
\hline De 0.401 a 0.60 & Moderado medio \\
\hline De 0.601 a 0.80 & Moderado alto \\
\hline De 0.801 a 1.00 & Alta influencia \\
\hline
\end{tabular}

Fuente: Elaboración propia.

La figura 1 muestra la influencia de los análisis de riesgos eléctricos con respecto a la reducción de accidentes eléctricos.

Los hallazgos obtenidos evidencian que la optimización de condiciones de trabajo, en base a un análisis de riesgos eléctricos, impacta directamente en la reducción de accidentes eléctricos, lo que implica mejoras para evitar la materialización de estos riesgos conocidos como arco y choque eléctrico.

Los análisis de arco y choque eléctrico se deben de realizar para diferentes actividades en las que una persona esté expuesta al peligro eléctrico; de acuerdo con el resultado estadístico, se tienen las correlaciones de 0.646 y 0.878 , respectivamente. Estas se interpretan como una influencia moderada alta y alta, con respecto a la reducción de accidentes; es por ello que, al realizar estos análisis, se tiene un gran aporte para evitar que los trabajadores se expongan a graves consecuencias ante este peligro.

En otras palabras, al gestionar y aplicar estos análisis, las variables de consecuencia y probabilidad disminuyen significativamente; por lo tanto, en caso llegase a suceder un accidente, ya sea por arco o por choque eléctrico, los daños en la persona serían menores o insignificantes. $\mathrm{Y}$, por otro lado, si se aplican de forma óptima las medidas de control, es muy raro o poco probable que este tipo de fenómenos se presente.

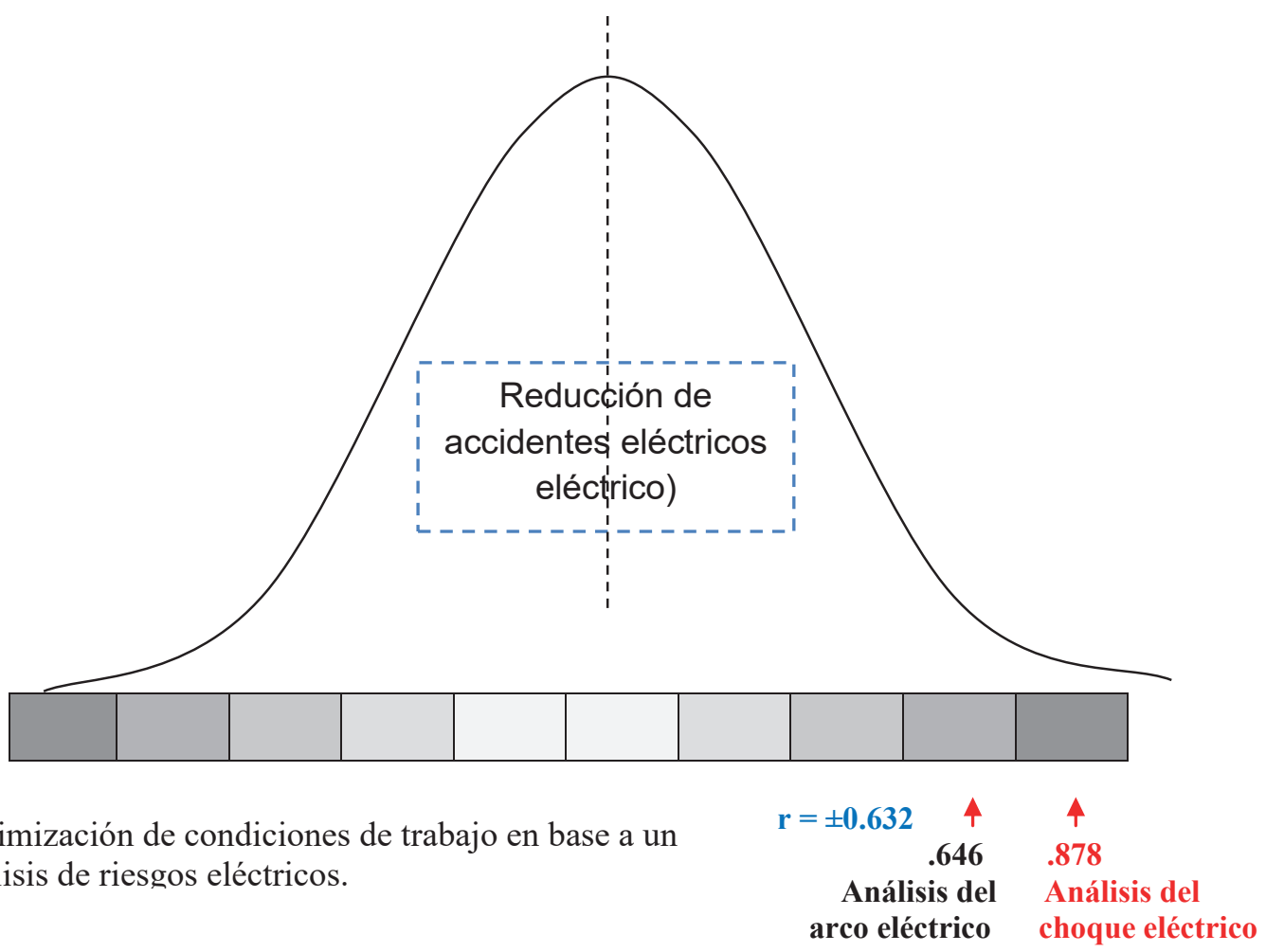

Figura 1. Correlación de Pearson. Fuente: Elaboración propia. 


\section{Análisis del arco eléctrico en sistemas eléctricos}

Un arco eléctrico está compuesto por dos etapas. La primera es el relámpago de arco, donde la exposición a este fenómeno se expresa en $\mathrm{cal} / \mathrm{cm}^{2}$, y la segunda es la ráfaga de arco, el cual es un fenómeno que se caracteriza por emitir ondas expansivas y por alcanzar elevadas temperaturas (pico de 25 $000{ }^{\circ} \mathrm{C}$ ). La energía del arco eléctrico llega a ionizar el aire liberando plasma y vapores de materiales conductores. Este fenómeno se genera por los cortocircuitos causados por el contacto entre conductores (falta de aislamiento entre estos), calentamiento del circuito, fallas de contactores para abrir o cerrar circuitos, materiales o herramientas que caen en líneas energizadas, aumento de tensión, daños en los equipos o malas maniobras o malas prácticas de los equipos.

Los efectos y consecuencias que puede causar un arco eléctrico llegan a ser muy críticos, desde lesiones leves o graves (quemaduras), incrustaciones, daños auditivos o en la retina y pérdida de la vida; adicionalmente, producen daños en las instalaciones.

\section{Calculo de arco eléctrico según IEEE 1584-2002}

Las ecuaciones empíricas son desarrolladas por el Institute of Electrical and Electronics Engineers (IEEE) con un equipo de trabajo sobre arco eléctrico. Los siguientes parámetros y resultados de pruebas se aplicaron bajo ciertas condiciones especiales (ver Tabla 5).

El cálculo de la energía incidente del arco eléctrico se realizó para los siguientes dos escenarios:

- Caso 1: tensión de 480 VAC, con 30 mm de distancia entre conductores, en un compartimento tipo caja y con un tiempo de 0.1 segundos. Como resultado se llegó a calcular una energía incidente de $5.12 \mathrm{cal} /$ $\mathrm{cm}^{2}$ a una distancia de alcance de $609 \mathrm{~mm}$ y una frontera de seguridad de $918 \mathrm{~mm}$.

- Caso 2: tensión de 4.16 kVAC, con 104 $\mathrm{mm}$ de distancia entre conductores, en un compartimento tipo caja y con un tiempo de 0.1 segundos. Como resultado se llegó a calcular una energía incidente de $1.24 \mathrm{cal} /$ $\mathrm{cm}^{2}$ a una distancia de alcance de $910 \mathrm{~mm}$ y una frontera de seguridad de $1352 \mathrm{~mm}$.

Para los casos anteriores se interpretó que, si aumenta la tensión y la distancia entre conductores, estos son inversamente proporcionales a la generación de energía incidente, y que la distancia de alcance de la energía incidente es directamente proporcional con respecto a las variables mencionadas.

\section{Prevención del arco eléctrico}

La única forma segura de prevenir el arco eléctrico es desenergizando el equipo antes de comenzar el trabajo, y, previo a ello, se debe de comprobar que esté realmente sin tensión. Aunque existen otros métodos para poder mitigar la severidad de este fenómeno, a continuación se presentan algunos ejemplos:

- El sistema de mitigación de relámpago de arco se genera al crear un sistema alterno de paso de corriente de baja impedancia, dirigiéndolo a un compartimento alterno controlado, lo que ocasiona que transfiera toda la energía en una dirección donde no se exponga al trabajador. Es decir, cuando se presenta un circuito con baja impedancia, el flujo de corriente tiene mayor tendencia a fluir por ese trayecto, ya que la ley de Ohm menciona que a menor resistencia aumenta la intensidad de corriente. De esta forma,

Tabla 5. Consideraciones a tomar para el uso de la norma IEEE 1584-2002.

\begin{tabular}{|c|c|c|c|}
\hline Parámetros & \multicolumn{3}{|c|}{ Rango de aplicación } \\
\hline Sistema de voltaje & 0.208 & 15 & $\mathrm{kV}$ \\
\hline Frecuencia & 50 & 60 & $\mathrm{~Hz}$ \\
\hline Falla sólida de corriente & 0.7 & 106 & $\mathrm{~mm}$ \\
\hline Espacio entre electrodos & 13 & \multicolumn{3}{|c|}{ Abierto, caja, MCC, panel, switchgear, cables } \\
\hline Tipo de compartimento & \multicolumn{3}{|c|}{ Tres fases } \\
\hline Sistema de tierra & \multicolumn{3}{|c|}{} \\
\hline Fases & \multicolumn{3}{|c|}{} \\
\hline
\end{tabular}

Fuente: National Fire Protection Association (2012). 
se logra direccionar la corriente de falla a un compartimento diseñado para soportar la liberación de la energía incidente y evitar daños a la persona.

- El sistema de dispositivos de protección limitador de corriente es un sistema convencional que cuenta con relés de protección, los cuales mandan una señal a un interruptor para abrir un circuito debido a una falla de corriente. En este caso, se desea implementar un sistema compuesto por relés con sensores de luz para emitir una señal más veloz al interruptor; así se evita la generación de un arco eléctrico. Cabe mencionar que la señal que se emite debe ser menor de 6 ciclos; asimismo, se conoce la existencia de dispositivos que interactúan desde 3 hasta 5 ciclos, pero el factor a decidir si se genera una condición eléctricamente segura es el tiempo para activar los dispositivos de protección.

- La electricidad circula en conductores de cobre, con el movimiento de electrones libres, mientras que los protones y neutrones se mantienen quietos y alcanzan una velocidad de hasta $1 \mathrm{~mm} / \mathrm{s}(0.001 \mathrm{~m} / \mathrm{s})$. Se piensa que la corriente es veloz simplemente porque los electrones libres que transfieren el flujo de corriente se encuentran por todo el circuito en una gran cantidad y en gran densidad; esta es la razón de la velocidad de la corriente. Se puede decir que por tal fenómeno se acerca a la velocidad de la luz, pero no es igual, ya que la velocidad de la luz tiene una constante de 299792458 m/s.

- En otras palabras, un relé de protección suele emitir una señal en 1 ciclo (0.016 s) y los interruptores demoran entre 3 a 5 ciclos, si se realiza la suma de ambos dispositivos. En el mejor de los casos se tiene una respuesta de
4 ciclos, mientras que en el peor, 6 ciclos. Si se tuviera la respuesta con un sensor de luz, es muy probable que se logre alcanzar hasta una respuesta de 2 ciclos, tiempo necesario para poder salvar más de una vida.

- Los equipos de protección personal (EPP) se tienen con los siguientes niveles de protección: $4,8,25$ y $40 \mathrm{cal} / \mathrm{cm}^{2}$, en las categorías $0,1,2,3$ y 4 . Estos son trajes completos para todo el cuerpo y deben cumplir con los requisitos mínimos de la norma.

En caso se requiera realizar algún trabajo expuesto a una fuente de energía activa, se tendrá que contar con una serie de controles, procedimientos, permisos y sumo cuidado para realizar la tarea.

Un análisis de relámpago de arco debe determinar la frontera de seguridad para un relámpago de arco, es decir, la energía incidente, la distancia segura de trabajo y el EPP que las personas deben utilizar dentro de la frontera.

\section{Análisis del choque eléctrico en sistemas eléctricos}

Cuando se habla del choque eléctrico, se hace referencia al riesgo de mayor conocimiento y sobre el cual se basa la mayoría de normas de seguridad.

El efecto que tiene la corriente sobre el cuerpo varía en cada caso. El rango-efecto que se conoce por el paso de la corriente a través del cuerpo se muestra en la Tabla 6.

Aunque la corriente de choque no pase a través de un órgano vital, de todas formas puede generar lesiones severas o la muerte por quemaduras internas (5 amperios o más); estas quemaduras pueden causar una muerte retardada, debido a que son internas y de tercer grado.

Tabla 6. Rango y efecto de la corriente.

\begin{tabular}{|c|c|}
\hline Corriente & Efecto \\
\hline $1 \mathrm{~mA}$ & Casi imperceptible \\
\hline $1-3 \mathrm{~mA}$ & Umbral de percepción (la mayoría de los casos) \\
\hline $3-9 \mathrm{~mA}$ & Sensación dolorosa \\
\hline $9-25 \mathrm{~mA}$ & Contracciones musculares (no se puede soltar) \\
\hline $25-60 \mathrm{~mA}$ & Parálisis respiratoria (puede ser fatal) \\
\hline $60 \mathrm{~mA}$ o más & Fibrilación ventricular (probablemente fatal) \\
\hline 4 A o más & Parálisis cardiaca (puede restablecerse mediante RCP) \\
\hline 5 A o más & Quemaduras de tejidos (fatal si daña órganos vitales) \\
\hline
\end{tabular}

Fuente: Industrial Technology Suppliers, Inc. (2014). 
Como se ha mencionado, la resistencia del cuerpo es un factor importante para poder determinar la cantidad de corriente que pasa a través del cuerpo.

\section{Calculo del choque eléctrico}

Si se desea realizar un cálculo para los casos mencionados, es necesario hacer referencia a la ley de Ohm cuando dice que la corriente va ser igual al nivel de voltaje sobre la resistencia.

Para el primer caso se tenía un equipo con un nivel de tensión de 480 VAC, al cual se aplicó la formula siguiente:

I=V/R . . I=480 Voltios / 1000 Ohm ...=0.48 Amp o $480 \mathrm{~mA}$

Para este caso se tuvo una resistencia de 1000 Ohm como valor referencial, la cual una persona normal tiene; es decir, bajo condiciones comunes de trabajo. Sin embargo, hay factores que producen variación en este último valor y por ello es variable la intensidad de corriente que logre atravesar el cuerpo.

Se podría decir que la mayoría de las muertes instantáneas producidas por un choque eléctrico están asociadas a la parálisis directa del sistema respiratorio (25 mA a más), a la detención del bombeo del corazón debido a una fibrilación ventricular $(60 \mathrm{~mA}$ a más) o a la detención inmediata y completa del corazón (4 amperios a más).

\section{Prevención del choque eléctrico}

Para todo trabajo o entorno con exposición a una fuente de energía activa, la mejor prevención es realizar el aislamiento de esta energía y drenar toda la presencia de energía residual. Sin embargo, se consideran como sistemas de protección a aquellos que evitan los contactos directos e indirectos. Estas son las categorías de protección:

- Protecciones completas: diseñadas para evitar cualquier tipo de contacto con las partes energizadas; por ejemplo, cubrir completamente las partes activas con un aislamiento capaz de soportar influencias externas. La protección debe contar con barreras y envolventes fijos, seguros $y$ robustos, brindando una calidad suficiente para asegurar los grados de protección requeridos.

- Protecciones parciales: diseñadas para evitar contacto accidental con las partes energizadas; es decir, las barreras y envolventes deben estar instalados a modo de un obstáculo duro.

- Medidas de protección complementarias: son los interruptores diferenciales de alta sensibilidad (IDn<30mA), cuya función es proteger al usuario en caso de contacto con el punto activo.

- Los equipos de protección personal dieléctricos, guantes dieléctricos (clases $00,0,1,2,3$ y 4 con resistencia de 500, 1000, 7500, 17000,26500 y 36000 VAC, respectivamente), zapatos dieléctricos y cascos dieléctricos.

No obstante, la debilidad del interruptor diferencial es que no actúa de forma preventiva, sino que se activa cuando ya existe un choque eléctrico.

Finalmente, mantener una distancia segura de aproximación a los conductores o partes de circuitos eléctricos energizados expuestos es un medio efectivo para conservar un ambiente eléctricamente seguro.

\section{CONCLUSIONES}

- Luego de realizar análisis sobre riesgos eléctricos, se concluye que el arco eléctrico es la liberación sin control de energía en dos etapas (relámpago y ráfaga) y el choque eléctrico es el paso de corriente a través del cuerpo; ambos son efectos bajo una condición subestándar y generan consecuencias mortales.

- En la evaluación del arco eléctrico se llegó a calcular que, para un nivel de tensión de 480 $\mathrm{V}$, la generación de energía incidente fue de $5.12 \mathrm{cal} / \mathrm{cm}^{2}$, a $918 \mathrm{~mm}$ de distancia segura; para un nivel de tensión de $4.16 \mathrm{kV}$, la generación de energía incidente fue de 1.24 $\mathrm{kV}$, a una distancia segura de352 $\mathrm{mm}$. Se interpreta que, a mayor tensión y distancia entre conductores, estos son inversamente proporcional a la generación de energía incidente y que la distancia de alcance de la energía incidente es directamente proporcional. En otras palabras, no necesariamente se interpreta que un mayor nivel de tensión equivale a que la energía incidente también sea mayor; por lo tanto, las medidas de control se deben implementar para equipos de baja y media tensión.

- En la evaluación del choque eléctrico, se realizó el análisis en base a la ley de Ohm, con un nivel de tensión de $480 \mathrm{~V}$ y una resistencia humana promedio de $1000 \mathrm{Ohm}$, 
y se obtuvo un paso de corriente de 480 $\mathrm{mA}$, el cual es suficiente para matar a una persona. Por ello, se reafirma que, cuanto más alta sea la resistencia de un cuerpo, la corriente será inversamente proporcional; es decir, se requiere una resistencia de 1667 Ohm como mínimo para evitar una parálisis respiratoria o la muerte.

- Después de evidenciar las consecuencias por exposición a riesgos eléctricos, el aporte en la prevención de riesgos eléctricos al sistema de gestión de SSO es la aplicación de la jerarquía de controles, implementando controles de ingeniería de protección, mitigación, identificación y aislamiento; adicional a ello, es importante la capacitación y concientización de los trabajadores, especialmente los del control administrativo, y el uso de equipos de protección personal específicos para realizar ciertas tareas.

\section{AGRADECIMIENTO}

Agradezco a mis padres por sus palabras y aliento incondicional.

\section{REFERENCIAS BIBLIOGRÁFICAS}

[1] Carlos, C. y Campos, F. (2013). Diagnóstico y reacondicionamiento de instalaciones eléctricas en operación. Electricidad. La revista energética de Nicaragua, 3(9), 22.

[2] Clavijo, R., Vera, V. y Landín, F. (2009). Análisis de los riesgos y peligros de incendio en estaciones de transformación de energía y centro de control de motores. (Tesis de grado). Escuela Superior Politécnica del Litoral, Ecuador.

[3] Industrial Technology Suppliers, Inc. (2014). Seguridad eléctrica alto voltaje en minería. Ciudad de Panamá, Panamá: Industrial Technology Suppliers, Inc.

[4] Institute of Electrical and Electronics Engineers (2002). IEEE 1584-2002. Guide for performing arc flash calculations. EE. UU.: Institute of Electrical and Electronics Engineers.

[5] López, J. (2003). Programa en Microsoft Visual Basic 6.0 para el análisis de riesgos eléctricos en oficinas y centros de cómputo. (Tesis de licenciatura). Universidad Nacional Mayor de San Marcos, Perú.
[6] Ministerio de Energía y Minas (28 de julio de 2016). Decreto Supremo n. ${ }^{\circ}$ 024-2016EM. Aprueban Reglamento de Seguridad y Salud Ocupacional en Minería. Recuperado de http://minem.gob.pe/_legislacionM. php?idSector=1\&idLegislacion=10221.

[7] Merino, J. (27 de marzo de 2013). Resolución Ministerial n. ${ }^{\circ}$ 111-2013-MEM-DM. Aprueban Reglamento de Seguridad y Salud en el Trabajo con Electricidad. Recuperado de https:// www.osinergmin.gob.pe/seccion/centro_ documental/PlantillaMarcoLegalBusqueda/ Resol-111-2013-MEM-DM.pdf.

[8] National Fire Protection Association (2012). NFPA 70E. Standard for electrical safety in the workplace. EE. UU.: National Fire Protection Association.

[9] Niebel, B. y Freivalds A. (2009). Ingeniería industrial. Métodos, estándares y diseño del trabajo. México D. F., México: McGraw-Hill.

[10] Rivadeneira, L. y Torres, V. (2010). Análisis de fallas y control de protecciones como prevención de riesgos eléctricos. (Tesis de grado). Escuela Superior Politécnica del Litoral, Ecuador.

[11] Serrano, J. (2011). Análisis de gestión de riesgos en el mantenimiento de un sistema eléctrico, caso de: una subestación de alta tensión. (Tesis de maestría). Instituto Politécnico Nacional, México.

[12] Sobrino, J. (2013) Hábitos para la mejora continua. Lima, Perú: Ojo Pródigo.

[13] Trejo, J. (2013). Comparación de la Norma NFPA 70E y la IEEE 1584-2002 para el análisis del arco eléctrico para el uso adecuado del equipo de protección personal. (Tesis de grado). Instituto Politécnico Nacional, México.

[14] Vásquez, R. (27 de marzo de 2017). La teoría de la causalidad de Frank Bird. Prevencionar. com. Recuperado de https://prevencionar. com/2017/03/27/la-teoria-la-causalidad-frankbird/. 


\section{Electrical safety in the workplace}

Frank Neyra Vela ${ }^{1}$

\begin{abstract}
Electricity is the driving force behind progress in all industry and globally, the most widely-used form of energy currently used; however, if it is not used properly and workers do not have the necessary training, there is potential for it to cause damage. This research studies the main hazards regarding electricity: electric arc and electric shock. For this reason, an electrical safety methodology that consists in recognizing the hazards associated with the use of electricity to take preventive actions to avoid hazards that cause injury or death is applied. After performing an analysis on electric arc and shock, results contributed to the prevention of accidents in the workplace, per the NFPA 70E and IEEE 1584-2002 standards.
\end{abstract}

Keywords: electrical safety; electric arc; electric shock.

\section{INTRODUCTION}

Nowadays, to interact with any electrical system, prior knowledge of the standards that establish the requirements for the diagnosis and evaluation of an electrical installation is required. This knowledge aims at identifying dangerous conditions, physical deterioration, misuse and other situations that increase the probability of risk to people and their property (Carlos \& Campos, 2013). Accordingly, there are safety requirements at the national level set forth in Supreme Decree No. 024-2016-EM, Reglamento de Seguridad y Salud Ocupacional en Minería [Regulations on Occupational Safety and Health in Mining] (Ministerio de Energía y Minas, July 28, 2016); and in Ministerial Resolution No. 111-2013-MEM-DM, Reglamento de Seguridad y Salud en el Trabajo con Electricidad [Regulations on Electrical Occupational Safety and Health] (Merino, March 17, 2013).

It is important to be aware of current electrical safety regulations, especially electric shock and arc hazards, which have existed since humankind began to use electric power. That being said, the number of deaths, serious injuries, burns and property losses has increased due to a lack of awareness about electrical safety (Industrial Technology Suppliers, Inc., 2014). Lack of awareness regarding electrical hazards is still currently a deficiency and can be solved by addressing the two initial stages of the conscious competence learning model: unconscious-incompetence and conscious-incompetence (Sobrino, 2013); in other words, workers who are not aware of the damage they could suffer and those who do not yet know how to work with electrical hazards. As a result of the lack of knowledge regarding electrical hazards, any worker in the industry is exposed to electrical hazards with the potential to cause serious damage. These hazards can be identified as substandard conditions. Currently, improvements in safety are conditioned by costs, company bureaucracy, commitment, executive management interests, and other reasons; unfortunately, it is often only after a worker is injured or dies that safer conditions for employees are adopted.

1 Industrial engineer. Currently working as Occupational Health and Safety engineer at the Compañía Minera Antapaccay. (Arequipa, Peru).

ORCID: https://orcid.org/0000-0002-1133-6875

E-mail: Frank.Neyra@glencore.com.pe 
Sobrino (2013) mentions that continuous improvement and its application can solve anticipated problems and help in the management of potential hazards, taking into account that it is a cycle that must be constantly repeated. Likewise, Niebel and Freivalds (2009) note that accidents are the result of a sequence of events caused by multiple reasons. On the other hand, Bird's accident causation theory attempts to neutralize the destructive effects of potential or actual losses, which are the consequence of unwanted events. Therefore, the correct application of a professional's knowledge and techniques to methods and work procedures can reduce the losses caused by such events (Vásquez, March 27, 2017).

Considering the above, the main objective of this article is to relate working conditions to electrical hazards; in addition, it seeks to enhance knowledge regarding exposure to electrical hazards in order to prevent accidents. Pursuant to those purposes, specific objectives are set to identify how a factor contributes to the energy intensity of an electric arc and shock, and to suggest a way to prevent or reduce accidents related to them. It should be noted that the purpose of this article is consistent with Bird's theory, as the causes and effects that cause accidents in the industry should be neutralized by the managing of hazards; thus, this research encourages the contribution of preventive solutions to avoid accidents related to electricity, including the use of personal protective equipment and technology that reduces the severity of hazards.

Finally, this study considers the contributions of other research related to electrical safety, such as that of López (2003), who designed a program in Microsoft Visual Basic 6.0 whose objective is to analyze electrical hazards that occur in offices and computer centers, through the identification of hazards and the proposal of corrective actions. Similarly, the study by Clavijo, Vera and Landín (2009), which helps to prevent fires by analyzing risks and hazards at energy transformation stations and engine control centers, is taken into account. In turn, Rivadeneira and Torres' thesis (2010) is also considered. They conducted a failure analysis and control on electrical risk protection components and proposed focusing on various protection elements to ensure the quality of an electrical installation, showing its main characteristics, as well as the use of mandatory personal protective equipment at work to save lives. In addition, the hazard analysis and management conducted by Serrano (2011) for the maintenance of an electrical system using the HAZOP methodology, designed to identify possible failures that could lead to economic, political, social and environmental problems, is also considered. Finally, Trejo's paper was also examined (2013). It compares the NFPA 70E and IEEE 15842002 standards and determines the workers' incident energy exposure, finding some criteria for an industrial power electrical system in which the magnitude of operating voltage and short-circuit current (electric arc) can harm people. In addition, hazard risk category is established according to the incident energy level and safety equipment (personal protective equipment) is identified to prevent accidents.

\section{METHODOLOGY}

This is a quantitative research project, as numerical measures and reports were used. The main techniques used were interview, observation and analysis of data or documentary research. See the description below:

- Interview was direct and was conducted on the maintenance personnel.

- Observation was direct and structured, because an observation (structured) guide, frequency list, checklist, anecdotal record and field journal were used.

- Documentary analysis considered a variety of sources as secondary data, such as previous research, books, reports, reports, guides, manuals, electronic documents and web pages, requirements for the implementation of an OHS management system (OHSMS) and national and international legal standards.

It is also explanatory, because a causal relationship was established; that is, not only was an attempt made to describe or approach a problem, but also to find its causes.

On the other hand, the unit of analysis corresponded to a group of workers of the electrical maintenance area. The measurement, subject of interest of this study, was conducted on this area.

In regard to the methodology used for the hazard analysis, a study of the possibilities and consequences of each electrical hazard (arc and electric shock) was conducted in such a way that working conditions can be optimized and risk can be maintained at the lowest level that is reasonably possible. For electric arc hazard, the National Fire Protection Association (2012) and the Institute of Electrical and Electronics Engineers (2002) establish calculation methods for incident energy and flash protection boundaries; whereas for electric shock, Ohm's law was considered to determine the passage of current through the body in different scenarios. 


\section{RESULTS}

Regarding the analysis and interpretation of results, a theoretical criterion of contrast was proposed for the general hypothesis, specific hypothesis 1 and specific hypothesis 2 , considering a $p$-value of 0.05 . Then, when $p>0.05$ is obtained, Ho will be accepted as valid. But, if $p<0.05$ is obtained, Ha will be accepted as valid. The Pearson correlation coefficient was used for the hypothesis contrast.

\section{General hypothesis}

The following association was posed regarding the general hypothesis:

- Ho: There is no relationship between electrical hazard analysis and working conditions and the reduction of electrical accidents.

- Ha: There is a relationship between electrical hazard analysis and working conditions and the reduction of electrical accidents.

Table 1. Contrast statistics of the general hypothesis.

\begin{tabular}{|c|c|c|}
\hline Model & $\mathbf{R}$ & Sig. F Change \\
\hline 1 & 0.885 & 0.005 \\
\hline
\end{tabular}

Source: Prepared by the author.

By means of SPSS21 software, a correlation of $R=$ 0.885 and a $p$-value of 0.005 were found (see Table 1). Thus, in accordance to the theoretical criterion, it was accepted that there is significant relationship between electrical hazard analysis and working conditions and the reduction of electrical accidents.

\section{Specific hypothesis 1}

The following association was posed regarding specific hypothesis 1 :

- Ho: There is no relationship between the nominal value of an electric arc and the reduction of electrical accidents.

- Ha: There is a relationship between the nominal value of an electric arc and the reduction of electrical accidents.

Table 2. Contrast statistics of the specific hypothesis 1.

\begin{tabular}{|l|l|l|}
\hline \multicolumn{3}{|c|}{ Electric_Arc_Analysis } \\
\hline Pearson correlation & Reduction_Electrical_Accident & 0.646 \\
\hline Sig. (1-tailed) & Reduction_Electrical_Accident & 0.022 \\
\hline No. & Reduction_Electrical_Accident & 10 \\
\hline
\end{tabular}

Source: Prepared by the author.
A correlation of $\mathrm{R}=0.646$ and a $p$-value of 0.022 were found (see Table 2). Thus, in accordance to the theoretical criterion, it was accepted that there is significant relationship between the nominal value of and electrical arc and the reduction of electrical accidents.

\section{Specific hypothesis 2}

The following association was posed regarding specific hypothesis 2 :

- Ho: There is no relationship between electric shock intensity and the reduction of electrical accidents.

- Ha: There is a relationship between electric shock intensity and the reduction of electrical accidents.

Table 3. Contrast statistics of the specific hypothesis 3.

\begin{tabular}{|l|l|l|}
\hline \multicolumn{3}{|c|}{ Electric_Shock_Analysis } \\
\hline Pearson correlation & Reduction_Electrical_Accident & 0.878 \\
\hline Sig. (1-tailed) & Reduction_Electrical_Accident & 0.000 \\
\hline No. & Reduction_Electrical_Accident & 10 \\
\hline
\end{tabular}

Source: Prepared by the author.

A correlation of $\mathrm{R}=0.878$ and a $p$-value of 0.000 were found (see Table 3). Thus, in accordance to the theoretical criterion, it was accepted that there is significant relationship between the intensity of an electric shock and the reduction of electrical accidents.

\section{DISCUSSION}

The hypotheses contrast is shown in Table 4 and Figure 1.

Table 4. Interpretation of Pearson's correlation ranges.

\begin{tabular}{|c|c|}
\hline Range & Interpretation \\
\hline from 0.000 to 0.20 & Weak influence \\
\hline from 0.201 to 0.40 & Weak-Moderate influence \\
\hline from 0.401 to 0.60 & Medium -Moderate influence \\
\hline from 0.601 to 0.80 & High-Moderate influence \\
\hline from 0.801 to 1.00 & High influence \\
\hline
\end{tabular}

Source: Prepared by the author.

The figure 1 shows the influence of electrical hazard analysis on the reduction of electrical accidents.

Findings show that the optimization of working conditions, based on an electrical hazard analysis, has a direct impact on the reduction of electrical accidents. 
This implies improvements that prevent the occurrence of electric arc and electric shock.

Electric arc and shock analyses must be performed for different activities in which a person is exposed to electrical hazards. According to the statistical result, correlations of 0.646 and 0.878 are obtained for electric arc and shock, respectively; these are interpreted as having a High and High-Moderate influence on accident reduction. Consequently, these analyses represent a major contribution to the prevention of hazards.

In other words, when managing and applying these analyses, the variables of consequence and probability decrease significantly; therefore, in the event of an accident, either by electric arc or shock, the person would suffer minimal or insignificant harm. On the other hand, if the control measures are properly applied, it is very rare or unlikely that this type of phenomenon will occur.

\section{Analysis of electric arc hazard in electrical systems}

An electric arc is composed of two stages. The first is arc flash, measured in cal $/ \mathrm{cm}^{2}$, and the second is arc blast, which is a phenomenon characterized by emitting shockwaves and by reaching high temperatures (peak temperature of $25,000^{\circ} \mathrm{C}$ ). The energy of the electric arc ionizes the air by releasing plasma and vapors from conductive materials. This phenomenon is the result of short circuits caused by the contact between conductors (due to the lack of insulation), heating of the circuit, failure of contactors to open or close circuits, materials or tools that fall on energized lines, power surges, equipment damage, misuse or malfunction.

The effects and consequences of an electric arc can be very critical, from minor to serious injuries (burns), incrustations, hearing or retinal damage and loss of life; in addition, they cause damage to facilities.

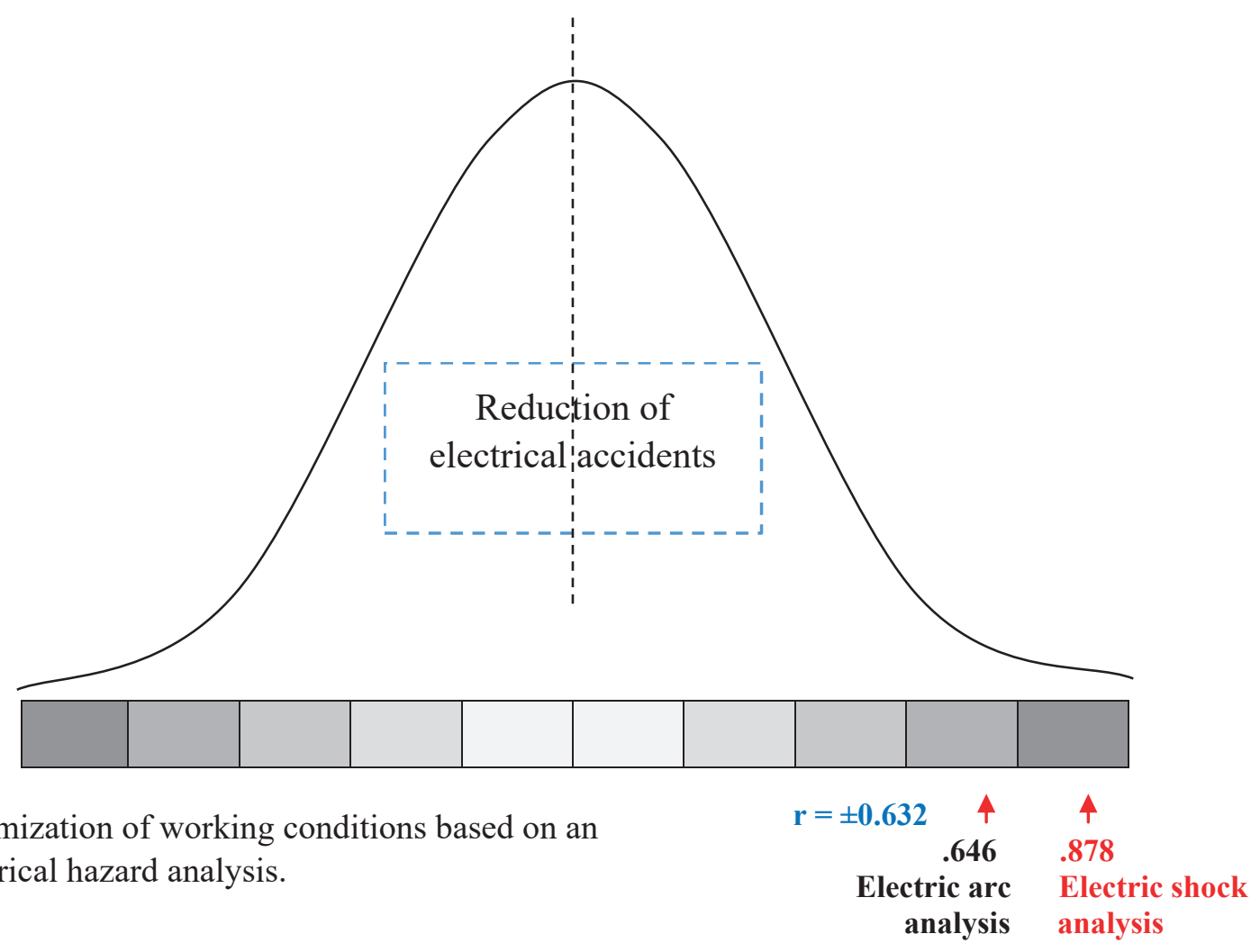

Figure 1. Pearson's correlation. Source: Prepared by the author. 


\section{Electric arc calculation according to IEEE 1584- 2002}

The Institute of Electrical and Electronics Engineers (IEEE) developed empirical equations for electric arc calculation. The following parameters and test results were applied under certain special conditions (see Table 5).

The incident energy of electric arc hazard was calculated for the following two scenarios:

- Case 1: 480 VAC voltage, with a 30 mm-gap between conductors, in a box enclosure and with a duration of 0.1 seconds. As a result, an incident energy value of $5.12 \mathrm{cal} / \mathrm{cm}^{2}$ was calculated at a working distance of $609 \mathrm{~mm}$ and a protection boundary of $918 \mathrm{~mm}$.

- Case 2: voltage of 4.16 kVAC, with a $104 \mathrm{~mm}$ gap between conductors, in a box enclosure and with a duration of 0.1 seconds. As a result, an incident energy value of $1.24 \mathrm{cal} / \mathrm{cm}^{2}$ was calculated at a working distance of $910 \mathrm{~mm}$ and a protection boundary of $1352 \mathrm{~mm}$.

For the above cases it was interpreted that, if the voltage and gap between conductors increases, these are inversely proportional to the incident power generation, and that the working distance of incident energy is directly proportional to the above variables.

\section{Electric arc hazard prevention}

The only safe way to prevent electric arc from occurring is to de-energize the equipment before working on it, and, before doing so, make sure equipment is really without voltage. Although there are other methods to reduce the severity of this phenomenon, here are some examples:
- An arc flash mitigation system results from the creation of an alternating low-impedance current system, which directs arc flash to a controlled alternate enclosure, therefore, preventing workers' exposure to hazard. That is, when there is a circuit with low impedance, current has a greater tendency to flow along that path, given that, as Ohm's law states, lower resistance increases current intensity. Thus, the fault current is directed to an enclosure designed to withstand the release of incident energy and prevent causing harm to people.

- The current limiting protection device system is a conventional system that has protective relays, which send a signal to a switch to open a circuit due to a current fault. In this case, the implementation of a system composed of relays with light sensors to emit a faster signal to the switch is intended; in this way, the generation of an electric arc is prevented. It should be mentioned that the emitted signal should last less than 6 cycles. Furthermore, there are devices that operate in 3 to 5 cycles. The time it takes to activate protective devices is the decisive factor in the creation of electrically safe conditions.

- Electricity flows through copper wires, where electrons move freely, while protons and neutrons remain still and reach a speed of up to $1 \mathrm{~mm} / \mathrm{s}(0.001 \mathrm{~m} / \mathrm{s})$. Current is thought to be fast because free electrons that transfer current flow are found throughout the circuit in large quantities and high densities, hence the speed of the current. Due to that phenomenon, it can be said that it approaches the speed of light, but it is not equal, as the speed of light is constant at 299792458 m/s.

Table 5. Considerations for the use of IEEE 1584-2002 standard.

\begin{tabular}{|c|c|c|c|}
\hline Parameters & \multicolumn{3}{|c|}{ Range } \\
\hline System voltage & 0.208 & 15 & $\mathrm{kV}$ \\
\hline Frequencies & 50 & 60 & $\mathrm{~Hz}$ \\
\hline Bolted fault current & 0.7 & 106 & $\mathrm{~mm}$ \\
\hline Gap between electrodes & 13 & 152 & Open air, box, MCC, panel, switchgear, cables \\
\hline Equipment enclosure type & \multicolumn{2}{|c|}{ Ungrounded, grounded, high resistance grounded } \\
\hline Grounding type & \multicolumn{3}{|c|}{ Three phase faults } \\
\hline Phases
\end{tabular}

Source: National Fire Protection Association (2012). 
- In other words, a protective relay usually emits a signal in 1 cycle (0.016 s) and switches operate in 3 to 5 cycles. If both devices' cycles are added together, there is a response in 4 cycles in the best-case scenario, and a response in 6 cycles in the worst. If a light sensor was used, a response in 2 cycles, the time needed to save more than one life, is very likely.

- Personal protective equipment (PPE) have protection levels of $4,8,25$ and $40 \mathrm{cal} / \mathrm{cm}^{2}$ for categories $1,2,3$ and 4 respectively. These are full body suits and must meet the standard minimum requirements.

In the event that work is required while in the presence of an active energy source, safety checks, procedures, permits and great care will be necessary to perform the task.

A flash arc hazard analysis will determine the arch flash protection boundary, i.e. the safe working distance, and the PPE that people must use within the boundary.

\section{Analysis of electric shock hazard in electrical systems}

Electric shock is the best-known electrical hazard on which most safety standards are based.

The effect of electric current on the body varies in each case. The effects of electric shock range, known as the current that passes through the body, are shown in Table 6.

Even when shock current does not pass through a vital organ, it can still lead to severe injury or death from internal burns (5 amperes or more); these burns can cause delayed death, because they are internal and third-degree burns.
As mentioned before, body resistance is an important factor in determining how much current passes through the body.

\section{Electric shock calculation}

To calculate electric shock hazard for the cases mentioned above, it is necessary to refer to Ohm's law that states that current will be equal to voltage divided by resistance.

In the first case, equipment with a voltage level of 480 VAC was used, to which the following formula was applied:

$\mathrm{I}=\mathrm{V} / \mathrm{R} \ldots \mathrm{I}=480$ Volts $/ 1000 \mathrm{Ohm} \ldots=0.48 \mathrm{Amp}$ or $480 \mathrm{~mA}$

In this case, a resistance of $1000 \mathrm{Ohm}$, the average human resistance under common working conditions, was used as reference value. However, there are factors that cause the variation of this last value and, therefore, the current intensity that passes through the body is also variable.

It could be said that most of the instantaneous deaths produced by an electric shock are associated with paralysis of the respiratory system ( $\geq 25$ $m A)$, heart failure due to ventricular fibrillation $(\geq 60$ $\mathrm{mA}$ ) or to immediate and complete heart arrest ( $\geq 4$ Amp).

\section{Electric shock hazard prevention}

For any work or environment with exposure to an active energy source, the best form of prevention is to isolate the energy source and drain all residual energy. Systems that avoid direct and indirect contacts are considered protection systems. These are the categories of protection:

Table 6. Effects of electric shock range.

\begin{tabular}{|c|c|}
\hline Current & Effect \\
\hline $1 \mathrm{~mA}$ & Barely perceptible \\
\hline $1-3 \mathrm{~mA}$ & Perception threshold (most cases) \\
\hline $3-9 \mathrm{~mA}$ & Painful sensation \\
\hline $9-25 \mathrm{~mA}$ & Muscular contraction (can't let go) \\
\hline $25-60 \mathrm{~mA}$ & Respiratory paralysis (may be fatal) \\
\hline $60 \mathrm{~mA}$ or more & Ventricular fibrillation (probably fatal) \\
\hline $4 \mathrm{~A}$ or more & Heart paralysis (probably fatal) \\
\hline $5 \mathrm{~A}$ or more & Tissue burning (fatal if vital organs are damaged) \\
\hline
\end{tabular}

Source: Industrial Technology Suppliers, Inc. (2014). 
- Comprehensive protective measures: Designed to avoid any kind of contact with energized parts; for example, completely cover the active parts with an insulation cover capable of withstanding external influences. The protection must have fixed, safe and robust barriers and enclosures, providing sufficient quality to ensure the required standards of protection.

- Partial protective measures: Designed to avoid accidental contact with energized parts; that is, barriers and enclosures must be installed as obstacles.

- Supplementary protective measures: Highsensitivity differential switches (IDn<30mA), whose function is to protect the user in the event of contact with the active area.

- Dielectric personal protective equipment, dielectric gloves (class 00, 0, 1, 2, 3 and 4 with maximum use voltage of $500,1000,7500,17$ 000, 26500 and 36000 VAC, respectively), dielectric shoes and dielectric helmets.

However, the downside of a differential switch is that it does not act preventively, but is activated when an electric shock is already occurring.

Finally, maintaining a safe approach distance from exposed conductors or parts of energized electrical circuits is an effective means of maintaining an electrically safe environment.

\section{CONCLUSIONS}

- After conducting analyses on electrical hazards, it is concluded that electric arc is the uncontrolled release of energy in two stages (arc flash and arc blast) and electric shock is the passage of current through the body; both phenomena can have deadly consequences when working in substandard conditions.

- In the electric arc hazard analysis, incident energy of $5.12 \mathrm{cal} / \mathrm{cm}^{2}$ was calculated for a voltage level of $480 \mathrm{~V}$ at a $918 \mathrm{~mm}$ arc boundary; incident energy of $1.24 \mathrm{kV}$ was calculated for a voltage level of $4.16 \mathrm{kV}$ at a $1352 \mathrm{~mm}$ arc boundary. It is interpreted that, when there is higher voltage and greater distance between conductors, they are inversely proportional to incident energy and that the working distance of incident energy is directly proportional. In other words, higher voltage does not necessarily mean higher incident energy; therefore, control measures should be implemented for low and medium voltage equipment.

- The electric shock hazard analysis was conducted based on Ohm's law. A voltage level of $480 \mathrm{~V}$ and an average human resistance of $1000 \mathrm{Ohm}$ were used for the calculation and a passing current of $480 \mathrm{~mA}$ was obtained, which is strong enough to kill a person. Therefore, it is reaffirmed that when the resistance of a body is higher, the current will be inversely proportional; that is, a resistance of at least $1667 \mathrm{Ohm}$ is required to avoid respiratory paralysis or death.

- After demonstrating the consequences of the exposure to electrical hazards, it was determined that the contribution for the prevention of electrical hazards to the OHS management system is the application of control hierarchy though the implementation of protection, mitigation, identification and isolation engineering controls. In addition, it is important to train and raise awareness among workers, particularly among those in management, and to use specific personal protective equipment to perform certain tasks.

\section{ACKNOWLEDGMENT}

To my parents, for their words and unconditional support.

\section{REFERENCES}

[1] Carlos, C. \& Campos, F. (2013). Diagnóstico y reacondicionamiento de instalaciones eléctricas en operación. Electricidad. La revista energética de Nicaragua, 3(9), 22.

[2] Clavijo, R., Vera, V. \& Landín, F. (2009). Análisis de los riesgos y peligros de incendio en estaciones de transformación de energía y centro de control de motores. (Degree thesis). Escuela Superior Politécnica del Litoral, Ecuador.

[3] Industrial Technology Suppliers, Inc. (2014). Seguridad eléctrica alto voltaje en minería. Panama City, Panama: Industrial Technology Suppliers, Inc.

[4] Institute of Electrical and Electronics Engineers (2002). IEEE 1584-2002. Guide for performing arc flash calculations. United States: Institute of Electrical and Electronics Engineers.

[5] López, J. (2003). Programa en Microsoft Visual Basic 6.0 para el análisis de riesgos eléctricos 
en oficinas y centros de cómputo. (Degree thesis). Universidad Nacional Mayor de San Marcos, Perú.

[6] Ministerio de Energía y Minas (July 21, 2016). Decreto Supremo n. ${ }^{\circ}$ 024-2016EM. Aprueban Reglamento de Seguridad y Salud Ocupacional en Minería. Retrieved from http://minem.gob.pe/_legislacionM. php?idSector=1\&idLegislacion=10221.

[7] Merino, J. (March 27, 2013). Resolución Ministerial n. ${ }^{\circ}$ 111-2013-MEM-DM. Aprueban Reglamento de Seguridad y Salud en el Trabajo con Electricidad. Retrieved from https:// www.osinergmin.gob.pe/seccion/centro documental/PlantillaMarcoLegalBusqueda/ Resol-111-2013-MEM-DM.pdf.

[8] National Fire Protection Association (2012). NFPA 70E. Standard for electrical safety in the workplace. United States: National Fire Protection Association.

[9] Niebel, B. \& Freivalds A. (2009). Ingeniería industrial. Métodos, estándares y diseño del trabajo. México D. F., Mexico: McGraw-Hill.
[10] Rivadeneira, L. \& Torres, V. (2010). Análisis de fallas y control de protecciones como prevención de riesgos eléctricos. (Degree thesis). Escuela Superior Politécnica del Litoral, Ecuador.

[11] Serrano, J. (2011). Análisis de gestión de riesgos en el mantenimiento de un sistema eléctrico, caso de: una subestación de alta tensión. (Master thesis). Instituto Politécnico Nacional, Mexico.

[12] Sobrino, J. (2013) Hábitos para la mejora continua. Lima, Peru: Ojo Pródigo.

[13] Trejo, J. (2013). Comparación de la Norma NFPA 70E y la IEEE 1584-2002 para el análisis del arco eléctrico para el uso adecuado del equipo de protección personal. (Degree thesis). Instituto Politécnico Nacional, Mexico.

[14] Vásquez, R. (March 27, 2017). La teoría de la causalidad de Frank Bird. Prevencionar. com. Retrieved from https://prevencionar. com/2017/03/27/la-teoria-la-causalidad-frankbird/. 